\title{
Mudanças na frequência do consumo de refeições em adolescentes residentes em área de vulnerabilidade social da região metropolitana do Rio de Janeiro, Brasil
}

\author{
Changes in meal frequency among adolescents living in a socially \\ vulnerable area of the Rio de Janeiro metropolitan region, Brazil
}

Rebecca de Almeida Maravalhas (https://orcid.org/0000-0001-9167-4382) ${ }^{1}$

Danilo Dias Santana (https://orcid.org/0000-0002-9769-7435) ${ }^{1}$

Rosana Salles-Costa (https://orcid.org/0000-0002-2307-4083) ${ }^{1}$

Gloria Valeria da Veiga (https://orcid.org/0000-0002-7985-0213) ${ }^{1}$

${ }^{1}$ Instituto de Nutrição Josué de Castro, Universidade Federal do Rio de Janeiro. Av. Carlos Chagas Filho 373, Bloco J, $2^{\circ}$ andar, Cidade Universitária. 21941-902 Rio de Janeiro RJ Brasil. dias.danilo@hotmail.com

\begin{abstract}
This study described changes in meal frequency over a 5-year period among adolescents living in the Rio de Janeiro metropolitan region. The data used were from two cross-sectional, population-based studies conducted by home visits. In 2005 the final sample was 1089 households with 511 adolescents (aged 12 to 18 years) and in 2010, 1121 households with 314 adolescents. Meal frequency was obtained through self-administered questionnaire and the adolescents were assessed for appropriate weight by BMI cut-off points, by sex and age group. Increasingly, traditional daily lunch was replaced by snacks (from $3.7 \%$ to $13.7 \%)$ and traditional dinner was eaten (62.9\% to $72.0 \%)$. Overweight adolescents ate breakfast less often than those not overweight (in 2005, $68.3 \%$ and $79.3 \%, p=0.02$ and, in 2010, 59.5\% and $77.4 \%, p=0.03$ ). Traditional daily lunch was increasingly replaced by snacks and consumption of traditional dinner increased over the 5-year period. Also, eating breakfast every day was associated with BMI classification at both study times: those who were overweight consumed breakfast less frequently.
\end{abstract}

Key words Meal frequency, Adolescents, BMI classification
Resumo O objetivo deste estudo foi descrever as mudanças na frequência do consumo de refeições de adolescentes residentes na região metropolitana do Rio de Janeiro, em período de 5 anos. Utilizaram-se dados de dois estudos transversais, de base populacional, que foram realizados por meio de visitas domiciliares. Em 2005, a amostra final foi de 1089 domicílios com 511 adolescentes (de 12 a 18 anos), e em 2010 de 1121 domicílios com 314 adolescentes. A frequência do consumo de refeições foi obtida por meio de questionário autopreenchido e a avaliação da adequação de peso dos adolescentes foi realizada com base nos pontos de corte do IMC por sexo e faixa etária. Houve aumento da substituição diária do almoço tradicional por lanche (3,7\% para 13,7\%) e no consumo do jantar tradicional (62,9\% para $72,0 \%)$. Os adolescentes com sobrepeso consumiram o desjejum com menor frequência do que aqueles sem sobrepeso (2005: $68,3 \%$ vs 79,3\% $p=0,02,2010: 59,5 \%$ vs $77,4 \% p=0,03)$. Conclui-se que houve aumento da substituição diária do almoço tradicional por lanche e no consumo do jantar tradicional no período de 5 anos. Ademais, a prática do desjejum diário se associou com a classificação de IMC nos dois momentos do estudo, de modo que aqueles com sobrepeso consomem o desjejum com menor frequência.

Palavras-chave Refeições, Adolescentes, Indice de massa corporal 


\section{Introdução}

$\mathrm{Na}$ adolescência são observadas algumas particularidades, como modificações no consumo e padrões alimentares e no estilo de vida ${ }^{1,2}$, que podem afetar a saúde do jovem e se perpetuar na vida adulta ${ }^{3,4}$. Estas modificações são preocupantes, pois têm sido associadas ao atual quadro epidemiológico, caracterizado pelo aumento expressivo de sobrepeso e obesidade em adolescentes de todo o mundo ${ }^{5,6}$, inclusive no $\mathrm{Brasil}^{7,8} \mathrm{e}$, ao desenvolvimento de doenças crônicas não transmissíveis nesta faixa etária4.

O consumo alimentar dos adolescentes caracteriza-se pelo excesso de alimentos hipercalóricos e industrializados, tipo fast foods, bebidas açucaradas e doces ${ }^{2,9,10}$, além do baixo consumo de alimentos fontes de fibras como frutas e hortaliças $^{11,12}$. A omissão das principais refeições, principalmente o desjejum ${ }^{13,14}$, ou a substituição por lanches frequentemente ricos em calorias, carboidratos e sódio ${ }^{12,15}$, também são traços do hábito alimentar na adolescência.

Neste contexto, o hábito de realizar as refeições mostra-se um fator de proteção ao sobrepeso e obesidade ${ }^{16,17}$, enquanto tais problemas de saúde têm sido associados à omissão das principais refeições ${ }^{18,19}$. Desta forma há uma baixa ingestão de alimentos considerados importantes para o desenvolvimento e saúde dos adolescentes, como alimentos fontes de cálcio que estão, em sua maioria, presentes no desjejum ${ }^{20,21}$, e alimentos marcadores de alimentação saudável presentes no almoço e jantar, como feijão, legumes ou verduras e frutas ${ }^{12}$, que dificilmente serão ingeridos em outro momento do dia.

Estudos sobre o consumo de refeições em adolescentes observaram que o desjejum é a refeição mais omitida, com maior frequência entre as meninas do que nos meninos ${ }^{15,22,23}$. Também já foi observado que o almoço é consumido mais frequentemente que o jantar ${ }^{18,24}$. Todavia, existem poucos relatos sobre como tais práticas tem se modificado ao longo do tempo e como podem se associar a sobrepeso e obesidade, particularmente no Brasil.

Em 2005, Estima et al..$^{25}$ em estudo transversal com adolescentes residentes em região cuja população é, predominantemente, de classe social mais baixa, observaram omissão de desjejum maior nas meninas $(12,4 \%)$ do que nos meninos $(4,5 \%)$ e elevada frequência de padrão de consumo insatisfatório de refeições (classificado quando não havia o consumo diário das três refeições principais como desjejum, almoço e jantar), na ordem de um terço dos adolescentes. Observaram também associação deste padrão insatisfatório de refeições com maiores médias de índice de massa corporal (IMC), particularmente nos meninos.

Em 2010, foi realizado outro estudo transversal na mesma localização do anterior (2005), no qual também foram obtidos dados referentes à frequência no consumo de refeições sendo possível investigar as mudanças temporais nessas práticas. Considerando o aumento na prevalência de sobrepeso observada neste período entre os dois estudos $^{26}$, tem-se como hipótese de que pode ter havido também aumento na omissão de refeições pelos adolescentes no período investigado e que este comportamento pode estar associado ao sobrepeso. Sendo assim, o objetivo deste estudo foi descrever as mudanças temporais na frequência do consumo de refeições e verificar a associação entre esta prática com variáveis socioeconômicas, demográficas e perfil de peso de adolescentes em período de 5 anos.

\section{Métodos}

\section{Amostragem}

Este trabalho utiliza dados de duas pesquisas de desenho transversal e de base populacional, realizadas por meio de visitas domiciliares, nas quais se investigaram residentes de Campos Elíseos, Duque de Caxias, no estado do Rio de Janeiro, Brasil. Na época do segundo estudo (2010), Campos Elíseos possuía população, predominantemente, urbana, estimada em 855.048 habitantes, que corresponde a $5,34 \%$ da população do estado $^{27}$. Em 2005, destacava-se por ser um dos distritos de menor renda per capitado município de Duque de Caxias, com apenas $26,8 \%$ das famílias com renda per capita acima de um salário mínimo, cerca de $83 \%$ das famílias classificadas nas classes sociais $\mathrm{C}$ e D, segundo a classificação da ABIPEME, e 52,3\% dos chefes de família não concluintes do ensino fundamental ${ }^{28}$.

A população de estudo corresponde aos adolescentes de 12 a 18,9 anos que fizeram parte do conjunto de famílias residentes em domicílios particulares permanentes (DPPs) de Campos Elíseos, nas datas de referência de cada pesquisa (2005 e 2010).

Em 2005 a amostra final foi de 1088 domicílios e 561 adolescentes elegíveis que foram entrevistados. Cinquenta adolescentes foram excluídos por inconsistência no preenchimento dos questioná- 
rios. Sendo assim, consideraram-se os dados de 511 adolescentes (taxa de resposta de 91,0\%). No segundo inquérito, realizado em 2010, a amostra final foi de 1121 domicílios, onde foram entrevistados 314 adolescentes, total dos considerados elegíveis. Nas duas pesquisas, foram considerados elegíveis os adolescentes com idade ente 12 e 18,9 anos, não portadores de deficiência física que impedisse a avaliação antropométrica e/ou responderem aos questionários e que não estivessem grávidas. As entrevistas domiciliares e aferições de medidas foram realizadas mediante assinatura do termo de consentimento livre e esclarecido pelo responsável do domicílio e só foram avaliados os adolescentes que aceitaram participar. Mais detalhes sobre os critérios de amostragem foram descritos em outros artigos publicados por SallesCosta et al. ${ }^{28}$ e Santana et al. ${ }^{26}$.

\section{Coleta de dados}

A coleta de dados, nos dois períodos, foi realizada por equipe de entrevistadores devidamente treinados para a função e ocorreu no período de abril a dezembro de 2005 (primeiro estudo) e de abril a dezembro de 2010 (segundo estudo).

Os questionários utilizados (2005 e 2010) foram elaborados, especificamente, para os estudos, utilizando perguntas já consolidadas em trabalhos semelhantes, ambos divididos em módulos, e similares nos dois momentos de modo a garantir a comparabilidade dos dados. Para o presente estudo foram utilizados: o modulo de identificação e controle com itens de 1 a 6 para caracterização do domicílio; o módulo de adolescentes com 24 questões sobre hábitos de vida e demográficas, das quais utilizaram-se aquelas referentes a frequência no consumo de refeições e raça do adolescente; o módulo de antropometria do qual utilizaram-se as medidas de massa corporal e estatura; módulo domicílio, com 6 tópicos, do qual utilizaram-se as questões referentes aos dados sociodemográficos como renda familiar mensal per capita e grau de escolaridade do chefe de família. Em 2005 foi verificada a escolaridade do adulto que respondeu ao questionário. Em 2010 foi avaliada a escolaridade do chefe da família já que esta prerrogativa foi exigida para ser o respondente do questionário. Acreditamos que esta diferença no método não implicou em viés nos resultados já que a maioria dos respondentes do questionário em 2005 era o chefe da família.

Os questionários passaram por pré-teste e estudo piloto com indivíduos com características semelhantes, mas que não participaram da pes- quisa, visando aperfeiçoamento do instrumento. Os questionários aplicados pelos entrevistadores foram revisados por um supervisor em breve espaço de tempo após a realização das entrevistas. Em caso de dúvida quanto ao correto preenchimento de alguma pergunta procurava-se esclarecimento junto ao morador através de contato por telefone ou, na impossibilidade deste contato, o entrevistador retornava ao domicílio para conferir a resposta.

A frequência do consumo de refeições foi investigada por meio das seguintes questões: em 2005 "Com que frequência você, usualmente, faz as seguintes refeições?”; em 2010 "Quantas vezes na semana você faz as seguintes refeições?”. Essas questões se referiram as principais refeições: desjejum, almoço e jantar tradicional e substituição do almoço ou jantar por lanches. As opções de respostas eram a) diariamente, b) 3 a 6 vezes por semana, c) 1 a 2 vezes por semana e d) nunca ou quase nunca.

Para a avaliação antropométrica, nas duas pesquisas todos os indivíduos foram avaliados vestindo roupas leves e descalços. O peso foi aferido com uma balança eletrônica portátil, de capacidade de até 150 quilogramas e variação de 50 gramas. A estatura foi medida por meio de estadiômetro portátil, com variação de 0,1 centímetros $(\mathrm{cm})$, sendo realizadas duas medidas e permitida uma variação máxima de $0,5 \mathrm{~cm}$ entre elas, calculando-se a média. $\mathrm{O}$ peso e a estatura foram obtidos seguindo as técnicas propostas por Gordon et al. ${ }^{29}$. O IMC foi calculado a partir do peso, em kg, dividido pela estatura, em metro, elevada ao quadrado.

As variáveis sociodemográficas investigadas foram: sexo, idade, cor da pele, renda familiar mensal per capita (rendimento total dividido pelo número de membros da família), expressa em múltiplos do salário mínimo ( R $300 \mathrm{em}$ 2005 e R\$ 510 em 2010) e grau de escolaridade de um adulto da família que respondeu o questionário (geralmente o chefe de família em 2005) e do chefe da família em 2010.

\section{Análise de dados}

A análise dos dados foi realizada utilizandose o software Statistical Program for the Social Sciences, versão 19,0 (SPSS, Chicago, IL), aplicando-se o procedimento Complex Sample para amostras complexas e expansão dos dados.

A frequência no consumo de refeições foi verificada por meio da proporção de adolescentes que consumiam as refeições desjejum, almoço e 
jantar (almoço e jantar considerados tradicionais com ingestão de comida ou almoço e jantar substituídos por lanche). Nas análises descritivas utilizaram-se as seguintes categorias: diariamente, 3 a 6 vezes na semana, 1 a 2 vezes na semana, nunca ou quase nunca. Nas análises de associação as frequências foram agrupadas em: consumo mais frequente (frequência diária e 3 a 6 vezes na semana) e menos frequente ( 1 a 2 vezes na semana, e nunca ou quase nunca).

O padrão de consumo de refeições foi avaliado como variável dicotômica (satisfatório ou insatisfatório), calculado por meio de escore pontuado de acordo com a frequência com que consumiam o desjejum, almoço e jantar (comida): diariamente $=0 ; 3$ a $6 \mathrm{x}$ semana $=1 ; 1$ a $2 \mathrm{x}$ semana $=2$; nunca ou quase nunca $=3$. Assim, a pontuação variou de zero a nove (a soma dos pontos atribuídos ao consumo de desjejum, almoço e jantar). Padrão de refeição satisfatório foi considerado como aquele em que o somatório alcançou valor igual a zero ou um, segundo Estima et al..$^{25}$.

A adequação de peso dos adolescentes foi calculada com base nos pontos de corte do IMC, por sexo e faixa etária, segundo critério da Organização Mundial de Saúde ${ }^{30}$. As categorias de baixo peso e adequado foram agrupadas em sem excesso de peso, e sobrepeso e obesidade em com sobrepeso. Sexo (masculino e feminino), idade (12 a 14,9 anos e de 15 a 18,9 anos) e cor da pele (branco e preto/pardo) foram analisados como variáveis binárias. Renda per capita foi apresentada nas categorias até $1 / 2$ salário mínimo, de $1 / 2$ a 1 salário mínimo e mais de 1 salário mínimo nas análises descritivas. Nas análises de associação utilizou-se a variável como binária (menor ou igual a $1 / 2$ salário mínimo e maior de $1 / 2$ salário mínimo). O grau de escolaridade de um adulto da família (2005) e do chefe de família (2010), foi apresentado nas análises descritivas nas seguintes categorias: A) Analfabeto/primeira etapa do ensino fundamental (EF) incompleto; B) primeira etapa do EF completo e segunda etapa do EF incompleto; C) EF completo/ensino médio incompleto; D), ensino médio completo e superior incompleto e completo. Nas análises de associação utilizaram-se as variáveis como binárias agrupando-se em $<8$ anos de estudo (agrupando-se as categorias A e B) e $\geq 8$ anos de estudo (agrupando-se as categorias $\mathrm{C}$ e D).

Inicialmente foi feita análise descritiva das variáveis do estudo, com as frequências e intervalos de confiança de 95\% (IC95\%) nos dois momentos (2005 e 2010). As frequências entre as pesquisas foram comparadas com base no teste qui-quadrado. Foi investigada a associação entre as variáveis dependentes (prática de fazer refeições: desjejum, almoço, jantar, almoço substituído por lanche e jantar substituído por lanche e padrão de refeições) com as variáveis independentes (renda per capita, grau de escolaridade do chefe da família, sexo, idade, cor da pele e perfil de peso), com base no teste qui-quadrado em cada período (2005 e 2010), aceitando-se valor de $\mathrm{p}<0,05$ para significância estatística. A magnitude das associações entre os períodos 2005 e 2010 foi avaliada pela odds ratio (OR) e IC95\%, e comparação destas ORs foi realizada por meio do método descrito por Altman e Bland ${ }^{31}$.

\section{Resultados}

Na Tabela 1 apresenta-se a distribuição dos adolescentes segundo características sociodemográficas e classificação de peso em 2005 e 2010. Houve diferença significativa entre os anos na classificação do peso $(\mathrm{p}=0,03)$, com diminuição no percentual de baixo peso (4,5\% para $0,9 \%$ ) e aumento do sobrepeso (16,3\% para 22,8\%).

Com relação à frequência na prática de refeições (Tabela 2) notou-se que no almoço-lanche a frequência de consumo diário aumentou de 3,7\% (2005) para $13,7 \%$ (2010) e o consumo nunca ou quase nunca diminuiu de $81,7 \%$ para $68,1 \%$ $(\mathrm{p}=0,005)$. Para o jantar-comida, houve um aumento no consumo diário de $62,9 \%$ (2005) para $72,0 \%(2010)(\mathrm{p}=0,002)$.

Observou-se ainda que, tanto em 2005 quanto em 2010, o consumo diário do desjejum se associou a classificação de peso, com maior prevalência entre os adolescentes sem sobrepeso do que entre aqueles com sobrepeso. $\mathrm{O}$ consumo diário do almoço-comida em 2005 se associou a idade, sendo mais prevalente nos mais novos do que nos mais velhos $(91,1 \%$ vs $84,1 \%, \mathrm{p}=0,04)$ (Tabela 3).

Em relação ao consumo diário do jantar-comida, em 2005 esta prática se associou a idade, sendo mais prevalente naqueles com idade $<15$ anos $(69,5 \%$ vs $57,6 \%, \mathrm{p}=0,005)$. Tanto os adolescentes mais velhos (>15 anos), quanto os de maior renda ( $>1 / 2$ salário mínimo), e assim como, os sem sobrepeso, tem aproximadamente 2 vezes mais chances de realizarem o jantar-comida diariamente em 2010 do que em 2005 (Tabela 3).

O padrão satisfatório de refeições se associou ao sexo e a idade em 2005 , sendo mais prevalente em meninos $(72 \%$ vs $62,2, \mathrm{p}=0,04)$ e nos ado- 
Tabela 1. Características sociodemográficas e classificação de peso com base no IMC dos adolescentes. Campos Elíseos, Duque de Caxias-RJ, 2005 e 2010.

\begin{tabular}{|c|c|c|c|c|c|c|}
\hline & \multicolumn{2}{|r|}{2005} & \multicolumn{2}{|r|}{2010} & \multirow{2}{*}{$\begin{array}{l}\text { Var. } \\
(\%)\end{array}$} & \multirow{2}{*}{$\begin{array}{l}\text { Valor } \\
\text { de } p^{1}\end{array}$} \\
\hline & $\mathbf{n}^{\star}$ & $\%(95 \% \mathrm{IC})$ & $\mathbf{n}^{\star}$ & $\%(95 \% \mathrm{IC})$ & & \\
\hline Sexo & 511 & & 314 & & & 0,668 \\
\hline Masculino & & $49,3(44,5-54,1)$ & & $51,0(44,3-57,7)$ & $+1,7$ & \\
\hline Feminino & & $50,7(45,9-55,5)$ & & $49,0(42,3-55,7)$ & $-1,7$ & \\
\hline Idade (anos) & 511 & & 314 & & & 0,178 \\
\hline $12-14.9$ & & $44,9(39,3-50,6)$ & & $50,3(42,7-57,9)$ & $+5,4$ & \\
\hline $15-18.9$ & & $55,1(49,4-60,7)$ & & $49,7(42,1-57,3)$ & $-5,4$ & \\
\hline Cor da pele & 510 & & 308 & & & 0,572 \\
\hline Preto/pardo & & $81,2(76,7-85,0)$ & & $78,9(70,9-85,1)$ & $-2,3$ & \\
\hline Branco & & $18,8(15,0-23,3)$ & & $21,1(14,9-29,1)$ & $+2,3$ & \\
\hline Renda per capita ${ }^{\star *}$ & 503 & & 304 & & & 0,470 \\
\hline Até $1 / 2$ salário mínimo & & $47,7(41,2-54,3)$ & & $50,5(40,7-60,2)$ & $+2,8$ & \\
\hline 1/2-1 salário mínimo & & $42,3(36,8-47,9)$ & & $36,5(28,5-45,3)$ & $-5,8$ & \\
\hline$>1$ salário mínimo & & $10,1(7,1-14,1)$ & & $13,1(7,4-22,1)$ & $+3,0$ & \\
\hline Grau de escolaridade ${ }^{* * *}$ & 509 & & 309 & & & 0,10 \\
\hline $\begin{array}{l}\text { Analfabeto/primeira etapa do EF } \\
\text { incompleto }\end{array}$ & & $18,5(14,9-22,8)$ & & $13,3(8,0-21,3)$ & $-5,2$ & \\
\hline $\begin{array}{l}\text { Primeira etapa do EF completo e segunda } \\
\text { etapa do EF incompleto }\end{array}$ & & $30,7(26,2-35,7)$ & & $26,8(19,9-35,1)$ & $-3,9$ & \\
\hline EF completo/EM incompleto & & $24,5(19,2-30,6)$ & & $36,1(27,9-45,1)$ & $+11,6$ & \\
\hline $\begin{array}{l}\text { EM completo e superior incompleto e } \\
\text { completo }\end{array}$ & & $26,3(21,6-31,5)$ & & $23,8(16,9-32,4)$ & $-2,5$ & \\
\hline Classificação do IMC & 511 & & 301 & & & 0,033 \\
\hline Baixo peso & & $4,5^{\#}(2,7-7,2)$ & & $0,9(0,3-2,8)$ & $-3,6$ & \\
\hline Peso adequado & & $72,2(66,9-76,9)$ & & $68,2(60,5-74,9)$ & $-4,0$ & \\
\hline Sobrepeso & & $16,3(12,7-20,6)$ & & $22,8^{\#}(17,3-29,5)$ & $+6,5$ & \\
\hline Obesidade & & $7,1(4,8-10,5)$ & & $8,1(5,1-12,8)$ & $+1,0$ & \\
\hline $\begin{array}{l}\text { * Os valores diferem devido a perdas em cada variável. } \\
\text { escolaridade de um adulto da família. EF: Ensino Fund } \\
\text { qui-quadrado }<0,05 \text {. }\end{array}$ & or & $\begin{array}{l}\text { alário mínimo: } 2 \\
\text { I: Ensino Médio. }\end{array}$ & & $\begin{array}{l}00 ; 2010=\mathrm{R} \$ 510 .{ }^{* *} \\
\text {-quadrado. } \text { TTeste de }\end{array}$ & ${ }^{*}$ Grau de & \\
\hline
\end{tabular}

lescentes com idade $<15$ anos $(73,6 \%$ vs $61,7 \%$, $\mathrm{p}=0,02)$. Em 2010 se associou à classificação de peso com maior prevalência naqueles sem sobrepeso $(75,4 \%$ vs $54,4 \%, \mathrm{p}=0,007)$ (Tabela 3$)$.

\section{Discussão}

Como resultados principais, destacam-se o aumento da substituição diária do almoço tradicional por lanche e o aumento do consumo do jantar tradicional, no período de 5 anos. Ademais, foi notada associação da prática do desjejum diário com a classificação de peso, nos dois momentos do estudo, de modo que aqueles com sobrepeso consomem o desjejum com menor frequência que aqueles sem sobrepeso. Esta associação também foi marcante em 2010, com maior frequência do padrão de refeições inadequado para aqueles com sobrepeso.

Com relação ao consumo de refeições, a maioria das pesquisas encontradas na literatura avaliou apenas o desjejum ${ }^{13,14}$. No presente estudo observou-se que a frequência de consumo diário desta refeição era maior naqueles sem sobrepeso. O mesmo foi observado por Legarrea et al..$^{32}$, com 21.385 estudantes adolescentes do Chile, no qual o consumo diário do desjejum foi mais comum para os indivíduos com peso adequado $(62 \%)$, e menos para os indivíduos com sobrepeso $(56,3 \%)$ e obesidade $(51,8 \%)$. Storey et al. ${ }^{19}$, em estudo com adolescentes canadenses, 
Tabela 2. Frequência do consumo de refeições e padrão de refeições dos adolescentes. Campos Elíseos, Duque de Caxias-RJ, 2005 e 2010.

\begin{tabular}{|c|c|c|c|c|c|c|}
\hline \multirow{2}{*}{ Refeições } & \multicolumn{2}{|r|}{2005} & \multicolumn{2}{|r|}{2010} & \multirow{2}{*}{ Var. (\%) } & \multirow{2}{*}{ Valor de $\mathbf{p}^{1}$} \\
\hline & $\mathbf{n}^{*}$ & $\%$ & $\mathbf{n}^{*}$ & $\%$ & & \\
\hline Desjejum & 509 & & 304 & & & 0,08 \\
\hline Todos os dias & & $76,7(71,8-81,0)$ & & $72,0(62,7-79,7)$ & $-4,7$ & \\
\hline 3 a 6 vezes por semana & & $7,1(4,6-10,7)$ & & $14,3(8,9-22,1)$ & $+7,2$ & \\
\hline 1 a 2 vezes por semana & & $7,1(4,8-10,2)$ & & $3,9(1,5-10,0)$ & $-3,2$ & \\
\hline Nunca ou quase nunca & & $9,2(6,4-13,1)$ & & $9,8(6,4-15,0)$ & $+0,6$ & \\
\hline Almoço-comida & 509 & & 305 & & & 0,44 \\
\hline Todos os dias & & $87,2(83,4-90,3)$ & & $86,7(80,2-91,3)$ & $-0,5$ & \\
\hline 3 a 6 vezes por semana & & $8,6(6,1-12,1)$ & & $8,6(5,2-13,9)$ & 0 & \\
\hline 1 a 2 vezes por semana & & $3,0(1,7-5,0)$ & & $1,6(0,5-4,8)$ & $-1,4$ & \\
\hline Nunca ou quase nunca & & $1,2(0,5-3,0)$ & & $3,1(1,0-9,5)$ & $+1,9$ & \\
\hline Almoço-lanche & 507 & & 287 & & & 0,005 \\
\hline Todos os dias & & $3,7(2,2-6,3)$ & & $13,7(7,5-23,7)$ & $+10,0$ & \\
\hline 3 a 6 vezes por semana & & $3,6(2,0-6,2)$ & & $7,1(3,7-13,2)$ & $+3,5$ & \\
\hline 1 a 2 vezes por semana & & $11,0(7,8-15,2)$ & & $11,1(5,7-20,5)$ & $+0,1$ & \\
\hline Nunca ou quase nunca & & $81,7(76,8-85,8)$ & & $68,1(57,6-77,0)$ & $-13,6$ & \\
\hline Jantar-lanche & 505 & & 285 & & & 0,29 \\
\hline Todos os dias & & $7,3(5,0-10,5)$ & & $15,5(10,2-22,8)$ & $+8,2$ & \\
\hline 3 a 6 vezes por semana & & $8,3(5,8-11,9)$ & & $5,0(2,4-9,9)$ & $-3,3$ & \\
\hline 1 a 2 vezes por semana & & $22,8(18,1-28,3)$ & & $18,1(11,7-27,0)$ & $-4,7$ & \\
\hline Nunca ou quase nunca & & $61,6(55,1-67,8)$ & & $61,4(51,2-70,7)$ & $+0,2$ & \\
\hline Jantar-comida & 509 & & 303 & & & 0,002 \\
\hline Todos os dias & & $62,9(57,0-68,5)$ & & $72,0(64,6-78,4)$ & $+9,1$ & \\
\hline 3 a 6 vezes por semana & & $20,3(16,1-25,2)$ & & $17,9(12,7-24,7)$ & $-2,4$ & \\
\hline 1 a 2 vezes por semana & & $7,8(5,2-11,5)$ & & $0,2(0,1-0,9)$ & $-7,6$ & \\
\hline Nunca ou quase nunca & & $9,0(6,2-12,9)$ & & $9,8(6,1-15,4)$ & $+0,8$ & \\
\hline Padrão de refeições & 509 & & 300 & & & 0,68 \\
\hline Satisfatório & & $67,0(61,8-71,9)$ & & $69,0(60,7-76,3)$ & $+2,0$ & \\
\hline Insatisfatório & & $33,0(28,1-38,2)$ & & $31,0(23,7-39,3)$ & $-2,0$ & \\
\hline
\end{tabular}

${ }^{\star}$ Os valores diferem devido a perdas em cada variável. ${ }^{1}$ Teste qui-quadrado.

Fonte: Autores.

observaram que aqueles sem sobrepeso, tiveram maior frequência de consumo de desjejum em comparação com os estudantes com sobrepeso e obesidade.

O padrão de refeições satisfatório também esteve associado à classificação de peso em 2010, sendo mais frequente naqueles sem sobrepeso, similar ao encontrado por Estima et al. ${ }^{25}$, com esta mesma população em 2005. Estes autores observaram que meninos com padrão de refeições insatisfatório (omitir regularmente pelo menos uma das três principais refeições) apresentaram maiores médias de IMC e de perímetro da cintura, do que aqueles com padrão satisfatório de refeições. O mesmo foi observado por Cnop et $a l .^{33}$, em estudantes de escolas públicas e parti- culares, nos quais hábitos irregulares de refeição foram associados com sobrepeso. Neste contexto, observamos que a omissão das principais refeições (desjejum, almoço e jantar), e não apenas do desjejum, pode estar relacionada ao sobrepeso e obesidade.

O aumento na prática do consumo diário do lanche em substituição ao almoço tradicional, encontrado no presente estudo, está em consonância com relatos de outros autores. Teixeira et al. ${ }^{15}$ notaram que um terço dos 106 alunos de uma escola técnica localizada em São Paulo, substituíram o almoço por lanche. Resultado similar foi encontrado por Araki et al. ${ }^{24}$, em estudo transversal com 71 adolescentes do ensino médio de escolas de São Paulo, no qual verificaram que 
Tabela 3. Associação entre consumo de refeições diárias e características sociodemográficas, razão de chances (Odds Ratio) e intervalos de confiança de 95\% (IC95\%) para magnitude das associações. Rio de Janeiro, Brasil, em 2005 e 2010.

\begin{tabular}{|c|c|c|c|c|c|c|c|c|}
\hline \multirow{2}{*}{ Variáveis } & \multicolumn{3}{|c|}{2005} & \multicolumn{3}{|c|}{2010} & \multirow{2}{*}{$\begin{array}{l}\text { Var. } \\
(\%)\end{array}$} & \multirow{2}{*}{$\begin{array}{c}\text { Odds ratio } \\
(95 \% \mathrm{IC})\end{array}$} \\
\hline & $\mathbf{n}^{*}$ & $\%$ & $\mathbf{p}^{1}$ & $\mathbf{n}^{\star}$ & $\%$ & $\mathrm{p}^{1}$ & & \\
\hline \multicolumn{9}{|l|}{ Desjejum } \\
\hline Sexo & & & 0,10 & & & 0,13 & & \\
\hline Masculino & 250 & 80,7 & & 156 & 76,0 & & $-4,7$ & $0,76(0,41-1,38)$ \\
\hline Feminino & 258 & 72,8 & & 147 & 67,6 & & $-5,2$ & $0,78(0,46-1,30)$ \\
\hline Idade (anos) & & & 0,35 & & & 0,22 & & \\
\hline $12-14.9$ & 228 & 79,1 & & 152 & 66,9 & & $-12,2$ & $0,53(0,28-0,99)$ \\
\hline $15-18.9$ & 280 & 74,7 & & 151 & 77,1 & & $+2,4$ & $1,13(0,63-2,05)$ \\
\hline Renda per capita ${ }^{\star *}$ & & & 0,43 & & & 0,81 & & \\
\hline$\leq 1 / 2$ salário mínimo & 238 & 78,1 & & 149 & 72,7 & & $-5,4$ & $0,74(0,45-1,23)$ \\
\hline >1⁄2 salário mínimo & 262 & 75,2 & & 144 & 70,9 & & $-4,3$ & $0,80(0,40-1,58)$ \\
\hline Grau de escolaridade ${ }^{* * *}$ & & & 0,86 & & & 0,66 & & \\
\hline$<8$ anos & 251 & 77,2 & & 121 & 69,2 & & $-8,0$ & $0,66(0,29-1,48)$ \\
\hline$\geq 8$ anos & 256 & 76,4 & & 178 & 73,4 & & $-3,0$ & $0,85(0,46-1,56)$ \\
\hline Classificação do IMC & & & 0,02 & & & 0,03 & & \\
\hline Sem sobrepeso & 389 & 79,3 & & 204 & 77,4 & & $-1,9$ & $0,89(0,54-1,46)$ \\
\hline Com Sobrepeso & 119 & 68,3 & & 90 & 59,5 & & $-8,8$ & $0,68(0,30-1,53)$ \\
\hline \multicolumn{9}{|l|}{ Almoço-lanche } \\
\hline Sexo & & & 0,83 & & & 0,66 & & \\
\hline Masculino & 250 & 3,5 & & 147 & 12,6 & & $+9,1$ & $3,93(1,27-12,20)$ \\
\hline Feminino & 256 & 4,0 & & 140 & 14,9 & & $+10,9$ & $4,23(1,13-13,57)$ \\
\hline Idade (anos) & & & 0,57 & & & 0,55 & & \\
\hline $12-14.9$ & 228 & 4,4 & & 142 & 18,6 & & $+14,2$ & $4,97(1,59-15,48)$ \\
\hline $15-18.9$ & 278 & 3,2 & & 145 & 8,9 & & $+5,7$ & $2,93(0,87-9,89)$ \\
\hline Renda per capita ${ }^{\star *}$ & & & 0,76 & & & 0,31 & & \\
\hline$\leq 1 / 2$ salário mínimo & 237 & 4,2 & & 146 & 17,8 & & $+13,6$ & $4,96(1,60-15,40)$ \\
\hline$>1 / 2$ salário mínimo & 262 & 3,5 & & 131 & 10,7 & & $+7,2$ & $3,12(0,73-13,36)$ \\
\hline Grau de escolaridade ${ }^{\star * *}$ & & & 0,48 & & & 0,17 & & \\
\hline$<8$ anos & 249 & 4,5 & & 118 & 10,0 & & $+5,5$ & $2,37(0,84-6,70)$ \\
\hline$\geq 8$ anos & 256 & 3,1 & & 165 & 16,7 & & $+13,6$ & $6,33(1,97-20,36)$ \\
\hline Classificação do IMC & & & 0,70 & & & 0,83 & & \\
\hline Sem sobrepeso & 387 & 4,0 & & 187 & 12,4 & & $+8,4$ & $3,41(1,19-9,75)$ \\
\hline Com Sobrepeso & 119 & 3,0 & & 89 & 13,5 & & $+10,5$ & $4,96(1,03-23,77)$ \\
\hline
\end{tabular}

$29 \%$ substituíam o almoço por lanches e, destes, $17 \%$ o faziam uma a duas vezes por semana. Em outra pesquisa, desenvolvida por Leal et al. ${ }^{23} \mathrm{com}$ 228 adolescentes de São Paulo, observou-se que 30,8\% deles também substituíam o almoço ou jantar por lanche. A substituição frequente do almoço por lanches, provavelmente, decorre de fatores relacionados a mudanças no estilo de vida dos adolescentes, influência da mídia ${ }^{34}$ e, possivelmente, dos comportamentos alimentares desordenados, que podem acarretar na diminuição da frequência do consumo das principais refeições ${ }^{35}$.
Ademais, o almoço com comida tradicional se associou à idade na primeira pesquisa, sendo a frequência de consumo diário maior entre os mais novos (12-14,9 anos). Isto pode, talvez, ser devido à influência que a família ainda exerce nos adolescentes mais jovens ${ }^{12}$. O consumo do jantar com comida tradicional também aumentou. Esta prática, talvez possa estar relacionada ao aumento do consumo do lanche em substituição ao almoço, ocasionando menor ingestão de comida tradicional ao longo do dia, provocando aumento na prática diária do jantar como um compor- 
Tabela 3. Associação entre consumo de refeições diárias e características sociodemográficas, razão de chances (Odds Ratio) e intervalos de confiança de 95\% (IC95\%) para magnitude das associações. Rio de Janeiro, Brasil, em 2005 e 2010.

\begin{tabular}{|c|c|c|c|c|c|c|c|c|}
\hline \multirow{2}{*}{ Variáveis } & \multicolumn{3}{|c|}{2005} & \multicolumn{3}{|c|}{2010} & \multirow{2}{*}{$\begin{array}{l}\text { Var. } \\
(\%)\end{array}$} & \multirow{2}{*}{$\begin{array}{c}\text { Odds ratio } \\
(95 \% \mathrm{IC})\end{array}$} \\
\hline & $\mathbf{n}^{\star}$ & $\%$ & $\mathbf{p}^{1}$ & $\mathbf{n}^{*}$ & $\%$ & $\mathrm{p}^{1}$ & & \\
\hline \multicolumn{9}{|l|}{ Almoço-comida } \\
\hline Sexo & & & 0,24 & & & 0,24 & & \\
\hline Masculino & 250 & 88,9 & & 158 & 89,6 & & $+0,7$ & $1,07(0,43-2,6)$ \\
\hline Feminino & 258 & 85,6 & & 146 & 83,6 & & $-2,0$ & $0,85(0,40-1,80)$ \\
\hline Idade (anos) & & & 0,04 & & & 0,97 & & \\
\hline $12-14.9$ & 228 & 91,1 & & 154 & 86,7 & & $-4,4$ & $0,63(0,26-1,5)$ \\
\hline $15-18.9$ & 280 & 84,1 & & 151 & 86,8 & & $+2,7$ & $1,24(0,60-2,57)$ \\
\hline Renda per capita ${ }^{* *}$ & & & 0,13 & & & 0,65 & & \\
\hline$\leq 1 / 2$ salário mínimo & 238 & 89,8 & & 149 & 88,3 & & $-1,5$ & $0,85(0,33-2,21)$ \\
\hline >1⁄2 salário mínimo & 262 & 84,5 & & 146 & 85,8 & & $+1,3$ & $1,11(0,49-2,49)$ \\
\hline Grau de escolaridade & & & 0,98 & & & 0,32 & & \\
\hline$<8$ anos & 250 & 87,2 & & 121 & 83,3 & & $-3,9$ & $0,73(0,31-1,68)$ \\
\hline$\geq 8$ anos & 256 & 87,2 & & 180 & 88,7 & & $+1,5$ & $1,16(0,50-2,69)$ \\
\hline Classificação do IMC & & & 0,54 & & & 0,61 & & \\
\hline Sem sobrepeso & 389 & 86,6 & & 204 & 87,3 & & $+0,7$ & $1,05(0,50-2,19)$ \\
\hline Com Sobrepeso & 119 & 89,1 & & 90 & 84,2 & & $-4,9$ & $0,64(0,21-1,91)$ \\
\hline \multicolumn{9}{|l|}{ Jantar-lanche } \\
\hline Sexo & & & 0,28 & & & 0,48 & & \\
\hline Masculino & 249 & 8,6 & & 146 & 17,5 & & $+8,9$ & $2,23(0,97-5,15)$ \\
\hline Feminino & 256 & 5,9 & & 138 & 13,4 & & $+7,5$ & $2,45(1,01-5,90)$ \\
\hline Idade (anos) & & & 0,14 & & & 0,66 & & \\
\hline $12-14.9$ & 227 & 5,1 & & 141 & 14,2 & & $+9,1$ & $3,10(1,14-8,4)$ \\
\hline $15-18.9$ & 277 & 9,1 & & 143 & 16,7 & & $+7,6$ & $2,01(0,95-4,24)$ \\
\hline Renda per capita ${ }^{* *}$ & & & 0,29 & & & 0,75 & & \\
\hline$\leq 1 / 2$ salário mínimo & 236 & 8,8 & & 144 & 14,9 & & $+6,1$ & $1,82(0,72-4,59)$ \\
\hline >1⁄2 salário mínimo & 260 & 5,8 & & 130 & 17,3 & & $+11,5$ & $3,36(1,35-8,33)$ \\
\hline Grau de escolaridade & & & 0,69 & & & 0,69 & & \\
\hline$<8$ anos & 248 & 7,8 & & 116 & 14,4 & & $+6,6$ & $1,97(0,84-4,65)$ \\
\hline$\geq 8$ anos & 255 & 6,7 & & 164 & 16,7 & & $+10,0$ & $2,78(1,09-7,06)$ \\
\hline Classificação do IMC & & & 0,59 & & & 0,29 & & \\
\hline Sem sobrepeso & 386 & 6,9 & & 187 & 12,2 & & $+5,3$ & $1,87(0,93-3,77)$ \\
\hline Com Sobrepeso & 118 & 8,5 & & 87 & 17,3 & & $+8,8$ & $2,26(0,80-6,36)$ \\
\hline
\end{tabular}

tamento de compensação pela má alimentação no período diurno.

Com relação ao sexo, como já visto na literatura, as meninas apresentaram frequência menor de consumo das principais refeições comparadas aos meninos, corroborando com os achados de Maia et al. ${ }^{11}$, nos quais, a adesão ao padrão não saudável, esteve associada às adolescentes do sexo feminino. Da mesma forma, Teixeira et al. ${ }^{15}$, observaram que mais meninas costumavam substituir refeições por lanches. Vieira et al. ${ }^{36}$ observaram que a ausência do jantar era cerca de três vezes mais prevalente também entre as meninas.

O padrão de refeições insatisfatório em 2005, também foi mais frequente nas meninas, e em 2010, esta associação se manteve, apesar de não ser significativa $(p=0,06)$. Isto pode estar relacionado ao fato de meninas apresentarem maior preocupação com o corpo, com a imagem corporal e a perda de peso, principalmente no início da adolescência, do que os meninos ${ }^{37}$, e com essa preocupação excessiva elas introduzem as práticas inadequadas de controle do peso, podendo ser ainda mais prejudicial a saúde ${ }^{38}$. Ressalta-se ainda 
Tabela 3. Associação entre consumo de refeições diárias e características sociodemográficas, razão de chances (Odds Ratio) e intervalos de confiança de 95\% (IC95\%) para magnitude das associações. Rio de Janeiro, Brasil, em 2005 e 2010.

\begin{tabular}{|c|c|c|c|c|c|c|c|c|}
\hline \multirow{2}{*}{ Variáveis } & \multicolumn{3}{|c|}{2005} & \multicolumn{3}{|c|}{2010} & \multirow{2}{*}{$\begin{array}{l}\text { Var. } \\
(\%)\end{array}$} & \multirow{2}{*}{$\begin{array}{c}\text { Odds ratio } \\
(95 \% \mathrm{IC})\end{array}$} \\
\hline & $\mathbf{n}^{*}$ & $\%$ & $\mathrm{p}^{1}$ & $\mathbf{n}^{*}$ & $\%$ & $\mathrm{p}^{1}$ & & \\
\hline \multicolumn{9}{|l|}{ Jantar-comida } \\
\hline Sexo & & & 0,12 & & & 0,55 & & \\
\hline Masculino & 250 & 67,0 & & 158 & 74,2 & & $+7,2$ & $1,41(0,78-2,55)$ \\
\hline Feminino & 258 & 59,0 & & 144 & 69,7 & & $+10,7$ & $1,59(0,87-2,90)$ \\
\hline Idade (anos) & & & 0,005 & & & 0,73 & & \\
\hline $12-14.9$ & 228 & 69,5 & & 154 & 73,3 & & $+3,8$ & $1,20(0,65-2,20)$ \\
\hline $15-18.9$ & 280 & 57,6 & & 148 & 70,7 & & $+13,1$ & $1,78(1,04-3,04)$ \\
\hline Renda per capita ${ }^{* *}$ & & & 0,83 & & & 0,19 & & \\
\hline$\leq 1 / 2$ salário mínimo & 238 & 63,4 & & 147 & 68,0 & & $+4,6$ & $1,22(0,69-2,17)$ \\
\hline$>1 / 2$ salário mínimo & 262 & 62,2 & & 146 & 76,4 & & $+14,2$ & $1,96(1,10-3,50)$ \\
\hline Grau de escolaridade ${ }^{\star \star *}$ & & & 0,77 & & & 0,57 & & \\
\hline$<8$ anos & 250 & 63,9 & & 119 & 69,6 & & $+5,7$ & $1,29(0,74-2,25)$ \\
\hline$\geq 8$ anos & 256 & 62,2 & & 180 & 73,2 & & $+11,0$ & $1,66(0,91-3,01)$ \\
\hline Classificação do IMC & & & 0,78 & & & 0,07 & & \\
\hline Sem sobrepeso & 389 & 63,3 & & 201 & 76,9 & & $+13,6$ & $1,92(1,10-3,37)$ \\
\hline Com Sobrepeso & 119 & 61,7 & & 91 & 60,8 & & $-0,9$ & $0,96(0,43-2,10)$ \\
\hline \multicolumn{9}{|l|}{$\begin{array}{l}\text { Padrão de refeição } \\
\text { satisfatório }\end{array}$} \\
\hline Sexo & & & 0,04 & & & 0,06 & & \\
\hline Masculino & 250 & 72,0 & & 156 & 75,7 & & $+3,7$ & $1,21(0,62-2,35)$ \\
\hline Feminino & 258 & 62,2 & & 143 & 61,8 & & $-0,4$ & $0,98(0,56-1,70)$ \\
\hline Idade (anos) & & & 0,02 & & & 0,52 & & \\
\hline $12-14.9$ & 228 & 73,6 & & 152 & 66,4 & & $-7,2$ & $0,71(0,36-1,39)$ \\
\hline $15-18.9$ & 280 & 61,7 & & 148 & 71,7 & & $+10,0$ & $1,57(0,88-2,78)$ \\
\hline Renda per capita ${ }^{\star *}$ & & & 0,41 & & & 0,29 & & \\
\hline$\leq 1 / 2$ salário mínimo & 238 & 68,9 & & 145 & 73,0 & & $+4,1$ & $1,21(0,66-2,21)$ \\
\hline$>1 / 2$ salário mínimo & 262 & 65,1 & & 144 & 64,5 & & $-0,6$ & $0,97(0,51-1,85)$ \\
\hline Grau de escolaridade ${ }^{\star * *}$ & & & 0,63 & & & 0,92 & & \\
\hline$<8$ anos & 250 & 66,0 & & 117 & 68,1 & & $+2,1$ & $1,10(0,51-2,36)$ \\
\hline$\geq 8$ anos & 256 & 68,2 & & 178 & 69,0 & & $+0,8$ & $1,03(0,56-1,89)$ \\
\hline Classificação do IMC & & & 0,53 & & & 0,007 & & \\
\hline Sem sobrepeso & 389 & 67,9 & & 201 & 75,4 & & $+7,5$ & $1,44(0,85-2,46)$ \\
\hline Com Sobrepeso & 119 & 64,3 & & 89 & 54,4 & & $-9,9$ & $0,66(0,33-1,30)$ \\
\hline
\end{tabular}

${ }^{*}$ Os valores diferem devido a perdas em cada variável; ${ }^{* *}$ Valor do salário mínimo: $2005=\mathrm{R} \$ 300 ; 2010=\mathrm{R} \$ 510 .{ }^{* *}$ Grau de escolaridade de um adulto da família. ${ }^{1}$ Teste qui-quadrado.

Fonte: Autores.

que a privação de uma ou mais refeições pode ser prejudicial, levando ao consumo exagerado em outras refeições ou a compulsão alimentar ${ }^{39,40}$.

Neste estudo não observamos associações significativas entre frequência de consumo de refeições com as variáveis sociodemográficas de cor da pele, renda e escolaridade. Todavia, tal investigação procede, considerando que a situação social e econômica da população dessa região é incoerente com a riqueza gerada no município, ressaltando a desigualdade na distribuição de renda e no acesso a bens e serviços, resultante da exclusão social. Tal situação pode comprometer as condições de alimentação ${ }^{28}$, podendo resultar em desfechos desfavoráveis a saúde dos adolescentes residentes desse município.

Os resultados deste estudo devem ser analisados de acordo com suas limitações e pontos fortes. Por ser realizado a partir de duas pesquisas transversais não permite determinar as relações 
de causalidade nas associações entre as variáveis estudadas. Acredita-se que futuros estudos longitudinais poderão contribuir no esclarecimento destas associações. $O$ fato de não ter investigado a composição nutricional dos lanches que substituem as refeições, não sendo possível detectar se são opções saudáveis ou não, também é uma limitação. Destaca-se como um dos pontos fortes a avaliação da mudança na frequência do consumo de refeições no tempo. Ademais, a avaliação da frequência no consumo de todas as refeições, e não somente do desjejum, também é um diferencial dos demais estudos sobre este tema em adolescentes. O fato de ter sido realizada essa avaliação em um contexto de população com ele- vada vulnerabilidade social, também fortalece o trabalho, pois há poucos estudos nesse contexto.

Conclui-se então que houve aumento da substituição diária do almoço tradicional por lanche e no consumo do jantar tradicional no período de 5 anos. Ademais, o consumo do desjejum diário se associou com a classificação de peso nos dois momentos do estudo, de modo que aqueles com sobrepeso consomem o desjejum com menor frequência. Estes resultados evidenciam a necessidade de orientação aos adolescentes quanto aos riscos à saúde advindos da omissão de refeições e de maior incentivo a práticas regulares de refeições para evitar o agravamento do perfil nutricional e os riscos que essas alterações podem causar.

\section{Colaboradores}

RA Maravalhas participou da concepção, análises estatísticas e redação do artigo. DD Santana participou da concepção, análises estatísticas e redação do artigo. R Salles-Costa participou da concepção do estudo e da redação final do artigo. GV Veiga participou da concepção do estudo, das análises estatísticas e redação final do artigo.

\section{Financiamento}

Conselho Nacional de Desenvolvimento Científico e Tecnológico (CNPq) (503139/2003-3 e 476344/2008-5) e Instituto Nacional de Câncer. 


\section{Referências}

1. Alfaris NA, Al-Tamimi JZ, Al-Jobair MO, Al-Shwaiyat NM. Trends of fast food consumption among adolescent and Young adult Saudi girls living in Riyadh. Food Nutr Res 2015; 59:26488.

2. Coelho SEAC, Gubert MB. Insegurança alimentar e sua associação com consumo de alimentos regionais brasileiros. Rev Nutr 2015; 28(5):555-567.

3. Assumpção D, Barros M, Fisberg R, Carandina L, Goldbaum M, Cesar C. Qualidade da dieta de adolescentes: estudo de base populacional em Campinas, SP. Rev Bras Epidemiol 2012; 15(3):605-616.

4. Silva DFO, Lyra CO, Lima SCVC. Padrões alimentares de adolescentes e associação com fatores de risco cardiovascular: uma revisão sistemática. Cien Saude Colet 2016; 21(4):1181-1196.

5. World Health Organization (WHO). Child and adolescent health and development: progress report 2009: highlights. Genebra: WHO; 2010.

6. Swinburn BA, Kraak VI, Allender S, Atkins VJ, Baker PI, Bogard JR, Brinsden H, Calvillo A, Schutter O, Devarajan R, Ezzati M, Friel S, Goenka S, Hammond RA, Hastings G, Hawkes C, Herrero M, Hovmand,PS Howden M, Jaacks LM, Kapetanaki AB, Kasman M Kuhnlein HV, Kumanyika SK, Larijani B, Lobstein T, Long MW, Matsudo VKR, Mills SDH, Morgan G, Morshed A, Nece PM, Pan A, Patterson DW, Sacks G, Shekar M, Simmons GL, Smit W, Tootee A, Vandevijvere S, Waterlander WE, Wolfenden L, Dietz WH. The Global Syndemic of Obesity, Undernutrition, and Climate Change: The Lancet Commission report. Lancet 2019; 393(10173):791-846.

7. Instituto Brasileiro de Geografia e Estatística (IBGE). Pesquisa de Orçamentos Familiares, 2008-2009: Antropometria e estado nutricional de crianças, adolescentes e adultos no Brasil. Rio de Janeiro: IBGE; 2010.

8. Brasil. Ministério da Saúde (MS). O Diagnóstico Alimentar e Nutricional e sua importância para o enfrentamento das Doenças Crônicas Não Transmissíveis. Sistema de Vigilância Alimentar e Nutricional - Boletim Epidemiológico [Internet]. Brasília: MS; 2019 [acessado 2020 out 21]. Disponível em: http://www.riocomsaude.rj.gov.br.

9. Enes CC, Camargo CM, Justino MIC. Ultra-processed food consumption and obesity in adolescents. Rev Nutr 2019; 32:e180170.

10. Monteiro LS, Hassan BK, Estima CCP, Souza AM, Verly Jr E, Sichieri R, Pereira RA. Consumo alimentar segundo os dias da semana - Inquérito Nacional de Alimentação, 2008-2009. Rev Saude Publica 2017; 51:93.

11. Maia EG, Silva LES, Santos MAS, Barufaldi LA, Silva SU, Claro RM. Padrões alimentares, características sociodemográficas e comportamentais entre adolescentes brasileiros. Rev Bras Epidemiol 2018; 21(Supl. 1):e180009.

12. Instituto Brasileiro de Geografia e Estatística (IBGE). Pesquisa Nacional de Saúde do Escolar - PENSE 2015. Rio de Janeiro: IBGE; 2016.

13. Barufaldi LA, Abreu GA, Oliveira JS, Santos DF, Fujimori E, Vasconcelos SML, Vasconcelos FAG, Tavares BM. ERICA: prevalência de comportamentos alimentares saudáveis em adolescentes brasileiros. Rev Saude Publica 2016; 50(Supl. 1):6s.
14. Trancoso SC, Cavalli SB, Proença RPC. Café da manhã: caracterização, consumo e importância para a saúde. Rev Nutr 2010; 23(5):859-869.

15. Teixeira AS, Philippi ST, Leal GVS, Araki EL, Estima CCP, Guerreiro RER. Substituição de refeições por lanches em adolescentes. Rev Paul Pediatr 2012; 30(3):330-337.

16. Wate JT, Snowdon W, Millar L, Nichols M, Mavoa H, Goundar R, Kama A, Swinburn B. Adolescent dietary patterns in Fiji and their relationships with standardized body mass index. Int J Behav Nutr Phys Act 2013; 10:45.

17. Arora M, Nazar GP, Gupta VK, Perry CL, Reddy KS, Stigler MH. Association of breakfast intake with obesity, dietary and physical activity behavior among urban school-aged adolescents in Delhi, India: results of a cross-sectional study. BMC Public Health 2012; 12:881.

18. Musaiger AO, Nabag FO, Al-Mannai M. Obesity, Dietary Habits, and Sedentary Behaviors Among Adolescents in Sudan: Alarming Risk Factors for Chronic Diseases in a Poor Country. Food Nutr Bull 2016; 37(1):65-72.

19. Storey KE, Forbes LE, Fraser SN, Spence JC, Plotnikoff RC, Raine KD, McCargar LJ. Adolescent Weight Status and Related Behavioural Factors: Web Survey of Physical Activity and Nutrition. J Obes 2012; 2012:342386

20. Peters BS, Verly E Jr, Marchioni DML, Fisberg M, Martini LA. The influence of breakfast and dairy products on dietary calcium and vitamin $\mathrm{D}$ intake in postpubertal adolescents and young adults. J Hum Nutr Diet 2012; 25(1):69-74.

21. Siega-Riz AM, Popkin BM, Carson T. Trends in breakfast consumption for children in the United States from 1965-1991. Am J Clin Nutr 1998; 67(4): 748S-756S.

22. Benedet J, Assis MA, Calvo MC, Andrade DF. Excesso de peso em adolescentes: explorando potenciais fatores de risco. Rev Paul Pediatr 2013; 31(2):172-181.

23. Leal GV, Philippi ST, Matsudo SM, Toassa EC. Consumo alimentar e padrão de refeições de adolescentes, São Paulo, Brasil. Rev Bras Epidemiol 2010; 13(3):457467.

24. Araki EL, Philippi ST, Martinez MF, Estima CCP, Leal GVS, Alvarenga MS. Pattern of meals eaten by adolescents from technical schools of São Paulo, SP, Brazil. Rev Paul Pediatr 2011; 29(2):164-170.

25. Estima CCP, Costa RS, Sichieri R, Pereira RA, Veiga GV. Meal consumption patterns and anthropometric measurements in adolescents from a low socioeconomic neighborhood in the metropolitan area of Rio de Janeiro, Brazil. Appetite 2009; 52(3):735-739.

26. Santana DD, Barros EG, Costa RSD, Veiga GV. Temporal changes in the prevalence of disordered eating behaviors among adolescents living in the metropolitan area of Rio de Janeiro, Brazil. Psychiatry Res 2017; 253:64-70.

27. Instituto Brasileiro de Geografia e Estatística (IBGE). Censo Demográfico 2010: Características da população e dos domicílios - Resultados do Universo. Rio de Janeiro: IBGE; 2011. 
28. Salles-Costa R, Pereira RA, Vasconcellos MT, Veiga GV, Marins VM, Jardim BC, Gomes FS, Sichieri R. Associação entre fatores socioeconômicos e insegurança alimentar: estudo de base populacional na Região Metropolitana do Rio de Janeiro, Brasil. Rev Nutr 2008; 21(Supl.):99s-109s.

29. Gordon CC, Chumlea WC, Roche AF. Stature, Recumbent Length and Weight. In: Lohman TG, Roche AF, Martorell R. Anthropometric Standardization Reference Manual. Champaign: Human Kinetics Books; 1988. p. 3-8.

30. Onis M, Onyango AW, Borghi E, Siyam A, Nishida C, Siekmann J. Development of a WHO growth reference for school-aged children and adolescents. Bull World Health Organ 2007; 85(9):660-667.

31. Altman DG, Bland JM. Interaction revisited: the difference between two estimates. BMJ 2003; 326(7382): 219.

32. Lopez-Legarrea P, Olivares PR, Almonacid-Fierro A, Gomez-Campos R, Cossio-Bolaños M, Garcia-Rubio $\mathrm{J}$. Association between dietary habits and the presence of overweight/obesity in a sample of 21,385 Chilean adolescents. Nutr Hosp 2015; 31(5):2088-2094.

33. Cnop ML, Monteiro LS, Rodrigues PRM, Estima CCP, Veiga GV, Pereira RA. Meal habits and anthropometric indicators in adolescents from public and private schools of the metropolitan region of Rio de Janeiro. Rev Nutr 2018; 31(1): 35-47.

34. Toral N, Conti MA, Slater B. Healthy eating according to teenagers: perceptions, barriers, and expected characteristics of teaching materials. Cad Saude Publica 2009; 25(11):2386-2394.

35. Matheson BE, Tanofsky-Kraff M, Shafer-Berger S, Sedaka NM, Mooreville M, Reina SA, Vannucci A, Shomaker LB, Yanovski SZ, Yanovski JA. Eating patterns in youth with and without loss of control eating. Int $J$ Eat Disord 2012; 45(8):957-961.
36. Vieira VCR, Priore SE, Ribeiro SMR, Franceschini SCC. Alterações no padrão alimentar de adolescentes com adequação pôndero-estatural e elevado percentual de gordura corporal. Rev Bras Saude Mater Infant 2005; 5(1):93-102.

37. Quiles-Marcos Y, Balaguer-Solá I, Pamies-Aubalat L, Quiles-Sebastián MJ, Marzo-Campos JC, Rodríguez-Marín J. Eating habits, physical activity, consumption of substances and eating disorders in adolescents. Span J Psychol 2011; 14(2):712-723.

38. Rodgers RF, McLean SA, Marques M, Dunstan CJ, Paxton SJ. Trajectories of Body Dissatisfaction and Dietary Restriction in Early Adolescent Girls: A Latent Class Growth Analysis. J Youth Adolesc 2016; 45(8):1664-1677.

39. Silva DCA, Frazão IS, Osório MM, Vasconcelos MGL. Percepção de adolescentes sobre a prática de alimentação saudável. Cien Saude Colet 2015; 20(11):32993308.

40. Vale AMO, Kerr LRS, Bosi MLM. Comportamentos de risco para transtornos do comportamento alimentar entre adolescentes do sexo feminino de diferentes estratos sociais do Nordeste do Brasil. Cien Saude Colet 2011; 16(1):121-132.

Artigo apresentado em 07/04/2020

Aprovado em 20/11/2020

Versão final apresentada em 22/11/2020

Editores-chefes: Romeu Gomes, Antônio Augusto Moura da Silva 